\title{
Avaliação do crescimento inicial das mudas de Tabebuia serratifolia (ipê amarelo) sob diferentes regimes hídricos
}

\author{
Marcos Cardoso Silva ${ }^{\mathrm{a}}$, Danilo Maciel ${ }^{\mathrm{a}}$, Maria Cristina Bueno Coelho ${ }^{\mathrm{a}}$, Marcos Giongo ${ }^{\mathrm{a}}$, \\ José Fernando Pereira ${ }^{a}$, André Ferreira dos Santos ${ }^{\mathrm{a}}$ \\ ${ }^{a}$ Universidade Federal do Tocantins, Brasil \\ *Autor correspondente (markuz@uft.edu.br)
}

\section{N F O}

\section{Keywords}

hydrical stress

germination

\section{Palavras-chaves} estresse hídrico germinação

\begin{abstract}
A B S T R A C T
Evaluation of the initial growth of Tabebuia serratifolia (ipê amarelo) seedlings under different water regimes.

The objective of this work was to evaluate the initial growth of Tabebuia serratifolia (Ipê Amarelo) seedlings under different water regimes, in order to find the one that best fits the species in the initial phase of growth. The following equipment was used to measure dendrometric variables, namely: digital caliper to measure DAC (Neck Height Diameter) and millimeter ruler to measure seedling height. The design used was a completely randomized design (DIC) where 6 replications were performed with 5 treatments in a total of 30 individuals, submitted to various water stress, and a control. It was concluded that the seeds of Ipê Amarelo have capacity to adapt to moderate water stress, presenting potential to be successfully used in some phases of degraded areas recovery; however, severe water deficit can affect the onset of germination and delay its development.
\end{abstract}

\section{R E S U M O}

Este trabalho teve como objetivo avaliar o crescimento inicial das mudas de Tabebuia serratifolia (ipê amarelo) sob diferentes regimes hídricos, de forma a encontrar o que melhor se adapta para a espécie em fase inicial de crescimento. Foram utilizados os seguintes equipamentos para a mensuração das variáveis dendrométricas sendo elas: paquímetro digital para medir DAC (Diâmetro Altura do Colo) e régua milimétrica para medir a altura das mudas. O delineamento utilizado foi DIC (Delineamento Inteiramente Casualizado) onde realizou-se 6 repetições com 5 tratamento num total de 30 indivíduos, submetidas a diversos estresse hídrico, e uma testemunha. Concluiu-se que as sementes de Ipê Amarelo apresentam capacidade de adaptação ao estresse hídrico moderado, apresentando potencial para ser utilizada com sucesso em algumas fases da recuperação de áreas degradadas; contudo, o déficit hídrico severo pode afetar o início da germinação e atrasar seu desenvolvimento. 


\section{INTRODUÇÃO}

A intensificação das atividades antrópicas tem acelerado a destruição dos ecossistemas e, consequentemente, a perda da biodiversidade no planeta, implicando não apenas na interrupção da integridade dos ciclos biológicos, como também, colocando em risco a própria sobrevivência humana (Batista, 2008).

A espécie Tabebuia serratifolia (ipê-amarelo) é uma espécie de ampla distribuição no território brasileiro, ocorrendo na região Amazônica, no Cerrado, na Caatinga e no Pantanal Mato-Grossense (Pott e Pott, 1994). A rica flora do Cerrado é constituída de inúmeras espécies, dentre elas está a espécie Tabebuia serratifolia, conhecida popularmente como ipê amarelo do cerrado. Dentre os gêneros que mais se destacam na família das Bignoniáceas, podem ser citados: Cybistax sp., Tabebuia sp. e Zeyheria sp. (Bittencourt Júnior, 2003).

Geralmente as espécies da Caatinga, Cerrado, entre elas a Tabebuia serratifolia, apresentam adaptações morfológicas e/ou fisiológicas que possibilitam a sobrevivência em condições de seca. A adaptação à falta de água durante vários meses do ano se mostra na forma, na cor, no metabolismo, nos ciclos vitais e organização social de todos os organismos desse bioma (MAIA, 2004). As plantas que habitam regiões de clima árido e semiárido apresentam várias estratégias para sobreviverem em condições de déficit hídrico.

A disponibilidade de água é tida como o principal fator limitante do potencial de estabelecimento das plantas, sobretudo no início do ciclo de vida (Nazário, 2006). Segundo Taiz e Zeiger (2004), o déficit hídrico pode ser definido como todo conteúdo de água de um tecido que está abaixo do conteúdo de água mais alto exibido no estado de maior hidratação.

Quando as plantas sofrem estresse hídrico, para evitar a desidratação essas fecham os estômatos reduzindo drasticamente a transpiração, já que a maior parte é transpiração estomática (Taiz e Zeiger, 2009). Além disso, certas plantas podem, juntamente com a redução do potencial hídrico do solo, promover uma maior redução no potencial da folha de forma a manter a diferença de potencial e continuar com os estômatos abertos por um maior período sem água. Essas plantas são comumente denominadas de plantas investidoras (LARCHER, 2000).

A tolerância da planta ao déficit hídrico é um importante mecanismo de resistência, garantindo o crescimento e a produção de biomassa em condições de baixa disponibilidade de água (GOMES et al., 2004).
Sendo assim, o presente estudo tem como objetivo avaliar o crescimento inicial (em altura e diâmetro) de mudas de Tabebuia serratifolia sob diferentes condições hídricas.

\section{MATERIAL E MÉTODOS}

O trabalho foi conduzido na área experimental da Universidade Federal do Tocantins (UFT), campus universitário de Gurupi, localizado na região sul do estado do Tocantins, compreendido pelas coordenadas $11^{\circ} 37^{\prime} 30^{\prime \prime}$ de latitude e $49^{\circ} 07^{\prime} 30^{\prime \prime}$ de longitude.

A caracterização climática local consiste em C2wA'a", clima úmido sub-úmido com moderada deficiência hídrica no inverno, evapotranspiração potencial média anual de $1.500 \mathrm{~mm}$, distribuindose no verão em torno de $420 \mathrm{~mm}$ ao longo dos três meses consecutivos com temperatura mais elevada (SEPLAN, 2012)

As sementes utilizadas que foram coletadas no início do mês de outubro de 2012 de apenas uma árvore matriz localizada na BR-153, no município de Gurupi (TO), foram armazenadas em um recipiente de vidro, local fresco para que não percam sua viabilidade que é baixa.

As sementes passaram por um processo de esterilização através da imersão em hipoclorito de sódio $1 \%$ por 30 segundos e enxágue em água destilada, por 30 segundos.

Foram utilizados os seguintes equipamentos para a mensuração das variáveis dendrométricas: paquímetro digital para medir o diâmetro à altura do colo (DAC) e régua milimetrada para medir a altura das mudas.

$\mathrm{O}$ delineamento utilizado foi inteiramente casualizado (DIC) onde realizaram-se 5 tratamentos com 6 repetições cada, totalizando 30 indivíduos, submetidos a diversos estresses hídricos, e uma testemunha (Figura 1).

Para realização da análise estatística das amostras dos DAC's das plântulas, foi desenvolvido um estudo da classe diamétrica, através do programa ASSISTAT. A tabela 1 apresenta os tratamentos testados no experimento e a respectiva disponibilidade hídrica para cada tratamento.

Tabela 1 - Diferentes tratamentos utilizados no experimento de desenvolvimento inicial de mudas de Tabebuia serratifolia.

\begin{tabular}{cc}
\hline TRATAMENTO & IRRIGAÇÃO \\
\hline T1 & $200 \mathrm{ml}$ \\
Tratamento 2 & $100 \mathrm{ml}$ \\
Tratamento 3 & $50 \mathrm{ml}$ \\
Tratamento 4 & $20 \mathrm{ml}$ \\
Tratamento 5 & $0 \mathrm{ml}$ \\
\hline
\end{tabular}


As figuras 1 e 2 apresentam como foram classificadas as mudas de cada tratamento levando em consideração a disponibilidade hídrica para cada tratamento.

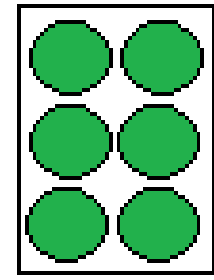

\section{$200 \mathrm{ml}$}

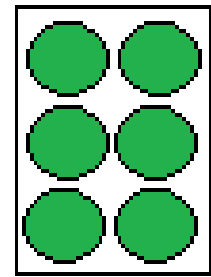

$100 \mathrm{ml}$

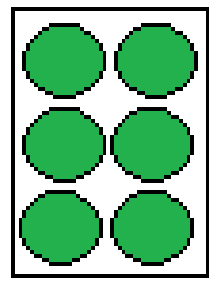

$50 \mathrm{ml}$

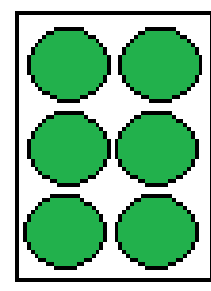

$20 \mathrm{ml}$

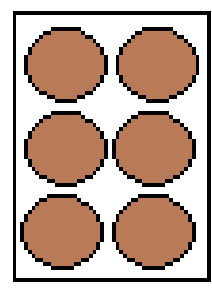

$0 \mathrm{ml}$

Figura 1 - Croqui demonstrativo dos tratamentos indicando as diferentes quantidades de água utilizada na irrigação das mudas.

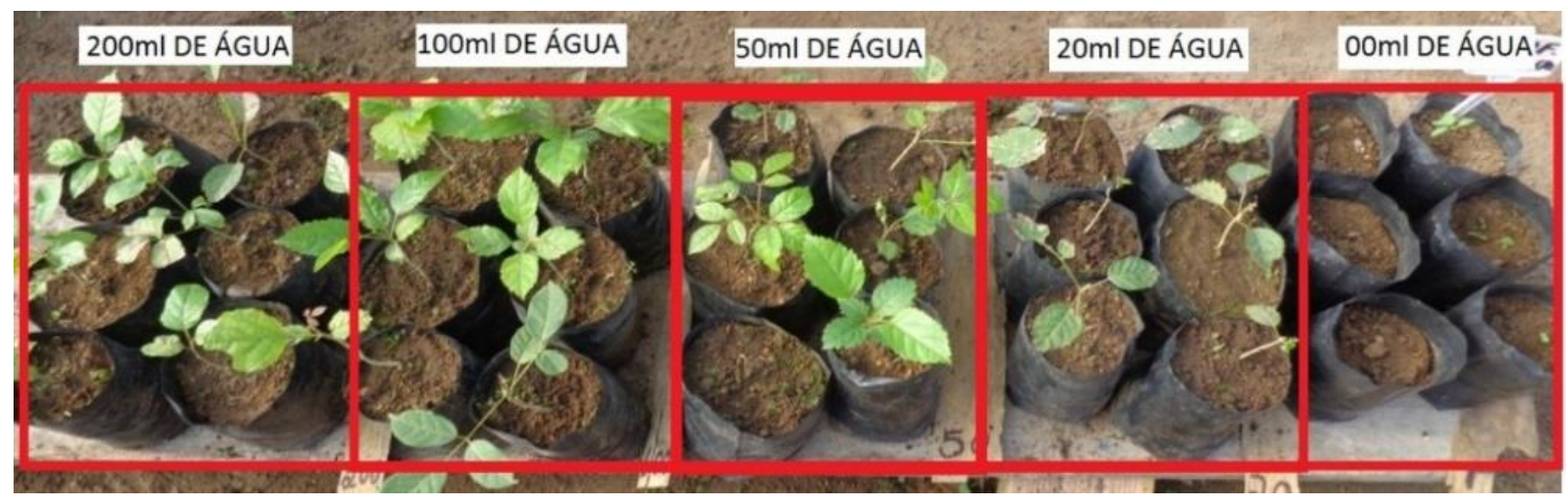

Figura 2 - Quantidade de água retida por tratamento.

O experimento foi desenvolvido em ambiente protegido em casa de vegetação onde o substrato era composto por: em terra de subsolo, esterco bovino, casca de arroz carbonizada na proporção $2: 1: 1$.

\section{RESULTADOS E DISCUSSÃO}

Os tratamentos 1,2 e 4 foram os que apresentaram melhores resultados, já o tratamento 3 uma semente não germinou, Nazário (2006), explica que muitas sementes mesmo após serem colocadas em condições favoráveis como temperatura, oxigênio e água, podem não germinar, assim como pode ser impedido devido à presença de um tegumento duro.

O tratamento 5 não recebeu nenhuma dosagem de água por ser a testemunha, de forma que as sementes das 6 repetições não germinaram por falta de condições favoráveis para o seu desenvolvimento.

Taiz e Zeiger (2009) relataram que o primeiro efeito mensurável do estresse hídrico é a diminuição no crescimento, causada pela redução da expansão celular. Além disso, essa redução na bio- massa seca, assim como no crescimento das plântulas, pode ser explicada pela diminuição no metabolismo das sementes, em função da menor disponibilidade de água para digestão das reservas e translocação de produtos metabolizados (Bewley e Black, 1994).

A tabela 2 apresenta a análise de variância para a variável altura (em centímetros) para as mudas de Tabebuia serratifolia.

Tabela 2 - Análise de variância para altura.

\begin{tabular}{lcccc}
\hline \multicolumn{1}{c}{ FV } & GL & SQ & QM & F \\
\hline Tratamentos & 4 & 389,6 & 97,4 & $35,1^{*}$ \\
Resíduo & 25 & 69,4 & 2,8 & \\
Total & 29 & 458,9 & & \\
\hline
\end{tabular}

* significativo ao nível de $5 \%$ de probabilidade $(\mathrm{p}<0.05)$

Pela análise de variância notamos que existe diferença significativa dos valores da altura do colo em função dos tratamentos utilizados, ou seja, indica que de acordo com a retenção de água existe maior ou menor estresse da muda. 
A tabela 3 apresenta os dados das alturas médias mensais (em centímetros) para as mudas de Tabebuia serratifolia.

Foi aplicado o Teste de Tukey ao nível de 5\% de probabilidade para avaliar a diferença estatística entre as médias de altura das mudas de Tabebuia serratifolia, como mostra a tabela 4 .

Tabela 3 - Média de altura mensal $(\mathrm{cm})$ por tratamento.

\begin{tabular}{ccccc}
\hline Tratamento & Jan. & Fev. & Mar. & Abr. \\
\hline 1 & 0 & 3 & 6,83 & 9,5 \\
2 & 0 & 4,6 & 7,3 & 11,3 \\
3 & 0 & 7,3 & 14,16 & 19,3 \\
4 & 0 & 6,5 & 13 & 16,83 \\
5 & 0 & 0 & 0 & 0 \\
\hline
\end{tabular}

Tabela 4 - Resultado do teste de Tukey $(\mathrm{p}<0,05)$ para a variável altura $(\mathrm{cm})$ no final do mês de abril.

\begin{tabular}{cc}
\hline Tratamento & Médias \\
\hline 1 & $4,80 \mathrm{~b}$ \\
2 & $5,83 \mathrm{~b}$ \\
3 & $10,21 \mathrm{a}$ \\
4 & $9,12 \mathrm{a}$ \\
5 & 0,00 \\
\hline
\end{tabular}

As médias seguidas pelas mesmas letras não diferem estatisticamente entre si, sendo assim as médias 1 e 2 são semelhantes estatisticamente, assim como as médias 3 e 4, que apresentaram melhores resultados para a variável altura, sendo considerados os melhores tratamentos já que apresentaram maiores médias de altura.

O tratamento 3 apresentou um melhor desenvolvimento, com altura média de 19,3 centímetros no quarto mês, em comparação ao $1^{\circ}$ e $2^{\circ}$ tratamento que recebeu menor quantidade de água $20 \mathrm{ml}$ e 50 $\mathrm{ml}$ respectivamente, as mudas não se desenvolveram bem chegando a 9,5 e 11,3 centímetros de altura.

O tratamento 1 se desenvolveu relativamente bem em comparação aos outros tratamentos, porém devido ao excesso de água utilizado na sua irrigação (200 ml), ele acaba sendo prejudicado havendo uma diminuição da velocidade de seu crescimento tendo altura máxima no final do projeto de 9,5 centímetros. $\mathrm{O}$ tratamento 5 (testemunha) não recebeu água e em consequência a sementes não germinaram.

Para a variável diâmetro também houve diferença significativa entre os tratamentos como pode ser observado na análise de variância na tabela 5 .
Tabela 5 - Análise de variância para a variável diâmetro a altura do colo $(\mathrm{cm})$ para mudas de Tabebuia serratifolia aos 4 meses (Fonte: autor).

\begin{tabular}{lcccc}
\multicolumn{1}{c}{ FV } & GL & SQ & QM & F \\
\hline Tratamento & 4 & 34,40 & 8,60 & $28,39^{*}$ \\
Resíduo & 25 & 7,57 & 0,30 & \\
Total & 29 & 41,97 & & \\
** significativo ao nível de $5 \%$ & de probabilidade $(\mathrm{p}<0.05)$
\end{tabular}

Pela análise de variância notou-se que houve diferença significativa dos valores do diâmetro altura do colo em função dos tratamentos utilizados, ou seja, indica que de acordo com a retenção de água existe maior ou menor estresse.

Para comparar os diferentes tratamentos foi realizado o teste de Tukey para comparar as médias dos tratamentos testados, como pode ser observado na tabela 6 .

Tabela 6 - Médias obtidas no teste Tukey $(\mathrm{p}<0,05)$ para a variável diâmetro à altura do colo $(\mathrm{cm})$.

\begin{tabular}{cc}
\hline Tratamento & Médias \\
\hline 1 & $2,08 \mathrm{~b}$ \\
2 & $2,08 \mathrm{~b}$ \\
3 & $3,25 \mathrm{a}$ \\
4 & $2,35 \mathrm{ab}$ \\
5 & $0,0 \mathrm{c}$ \\
\hline
\end{tabular}

De acordo com o teste Tukey, o tratamento 3 obteve os melhores resultados para a variável diâmetro à altura do colo, sendo semelhante estatisticamente com o tratamento 4 . Nota-se uma relação do estresse hídrico com o desenvolvimento das mudas. As testemunhas não germinaram visto que necessitam de água, e dentre os tratamentos o melhor foi o 3 pois mostrou maior desenvolvimento das mudas em altura e diâmetro.

\section{CONCLUSÕES}

As sementes de ipê amarelo apresentam capacidade de adaptação ao estresse hídrico moderado, apresentando potencial para ser utilizada com sucesso em algumas fases da recuperação de áreas degradadas; contudo, o déficit hídrico severo pode afetar o início da germinação e atrasar seu desenvolvimento.

O tratamento 3 apresentou melhor crescimento em altura e diâmetro utilizando $50 \mathrm{ml}$ de água na irrigação, indicando que o ipê amarelo se desenvolve bem com pequenas quantidades de água, sendo indicada para recuperação de áreas degradadas. 


\section{REFERÊNCIAS BIBLIOGRÁFICAS}

BATISTA, C.U.N. et al. Tolerância à inundação de Cecropia pachystachya Trec. (Cecropiaceae): aspectos ecofisiológicos e morfoanatômicos. Acta Botânica Brasilica. São Paulo, v. 22, n.1, p.91-98, jan/mar 2008.

BEWLEY, J.; BLACK, M. Seeds: physiology of development and germination. 3.ed. New York: Plenum Press, 1994. 445p.

LARCHER, W. Ecofisiologia vegetal. São Paulo: Rima Artes e Textos, 2000. 531p.

BITTENCOURT JÚNIOR, N.S. Auto-incompatibilidade de ação tardia e outros sistemas reprodutivos em Bignoniaceae. 2003. 275f. Tese (Doutorado em Biologia Vegetal). Universidade de Campinas.

GOMES, M.M.A. et al. Interactions between leaf water potential, stomatal conductance and abscisic acid content of orange trees submitted to drought estresse. Brazilian Journal of Plant Physiology, Londrina, v.16, n.3, p.155-161, Sept./Dec.2004.

MAIA, G.N. Caatinga: árvores e arbustos e suas utilidades. São Paulo: D\&Z computação gráfica e editora, 2004. 413p.

NAZÁRIO, P. Tratamento pré-germinativo visando minimizar a dormência em sementes de tucumã (Astrocaryum aculeatum G. Mey. 2006. 89f. Dissertação (Mestrado) Instituto Nacional de Pesquisas da Amazônia, Manaus, 2006.

POTT, A.; POTT, V.J. Plantas do Pantanal. Corumbá: EMBRAPA/CPAP - SPI, 1994. 320p.

SEPLAN, Secretaria do Planejamento e da Modernização da Gestão Pública. Atlas do Tocantins: subsídios ao planejamento da gestão territorial. Palmas, TO. 6. ed.80. 16 a 31 p. 2012.

SILVA, E.C. et al. Stomatal changes induced by intermittent drought in four umbu tree genotypes. Brazilian Journal of Plant Physiology, Londrina, v.21, n.1, p.33-42, Jan./Mar. 2009.

TAIZ, L.; ZEIGER, F. Fisiologia vegetal. 3.ed. Porto Alegre: Artmed, 2004. p.719.

TAIZ, E.; ZEIGER, L. Fisiologia vegetal. 4.ed. Porto Alegre: ARTMED, 2009. 848p. 